\title{
The production of germanium in asymptotic giant branch stars
}

\author{
Amanda Karakas ${ }^{* i}$ \\ Origins Institute and the Department of Physics \& Astronomy, McMaster University Hamilton \\ ON L8S 4M1, Canada \\ E-mail: karakas@physics.mcmaster.ca
}

\section{Maria Lugaro}

Astronomical Institute, Princetonplein 5, 3584 CC Utrecht, The Netherlands

E-mail: M.Lugaro@phys.uu.nl

\section{Roberto Gallino}

Dipartimento di Fisica Generale, Università di Torino, Via P. Giuria 1, I-10125 Torino, Italy

E-mail: gallino@ph.unito.it

\begin{abstract}
Observations of planetary nebulae by Sterling, Dinerstein and Bowers have revealed enhancements in the element Germanium (Ge) by factors of 3 to 10 compared to solar. Germanium can be produced by slow-neutron capture (s-process) in asymptotic giant branch (AGB) stars. We compute the detailed nucleosynthesis of a series of AGB models of various masses (1.9 to $6.5 \mathrm{M}_{\odot}$ ) and metallicities from the zero-aged main sequence to near the end of the thermally-pulsing AGB phase. We include a partial mixing zone of constant mass at the deepest extent of each dredgeup episode. During the interpulse period this mixing zone will produce a ${ }^{13} \mathrm{C}$ pocket in the top $\sim 1 / 10^{\text {th }}$ of the He-rich intershell, releasing free neutrons that can be captured by iron-seed nuclei. Using a post-processing code that includes 156 species, 1260 reaction rates and time-dependent convective mixing, we follow the nucleosynthesis of all stable isotopes up to ${ }^{75} \mathrm{As}$, including the five stable Ge isotopes. The surface Ge abundance at the end of the evolution was not enhanced in models with no partial mixing i.e. $[\mathrm{Ge} / \mathrm{Fe}] \approx 0$, except the $6.5 \mathrm{M}_{\odot}$ models which had $[\mathrm{Ge} / \mathrm{Fe}]$ $\approx 0.2$. Models with partial mixing produced surface enhancements of at most a factor of 3.6, i.e. $[\mathrm{Ge} / \mathrm{Fe}] \lesssim 0.5$ except the $2.5 \mathrm{M}_{\odot}, Z=0.004$ model with $[\mathrm{Ge} / \mathrm{Fe}]=0.77$. Given the difficulties in matching the compositions of the most Ge-enriched PNe we suggest a late thermal pulse mixed pure He-intershell material, enriched in $\mathrm{Ge}$, with the small amount of remaining convective envelope.
\end{abstract}

International Symposium on Nuclear Astrophysics - Nuclei in the Cosmos - IX

June 25-30 2006

CERN, Geneva, Switzerland

\footnotetext{
*Speaker.

${ }^{\dagger}$ H. G. Thode Fellow at the Origins Institute
} 


\section{Introduction}

After the thermally-pulsing asymptotic giant branch (TP-GB) phase is terminated, low to intermediate mass stars $\left(0.8\right.$ to $\left.8 \mathrm{M}_{\odot}\right)$ evolve through the planetary nebulae $(\mathrm{PNe})$ phase before finally ending their lives as white dwarfs [1]. The gaseous nebula is the remnant of the deep convective envelope that once surrounded the core, which is now exposed as the central star of the PN. Thus the abundances of the nebula should reveal information about the chemical processing that took place during the AGB; and more precisely, information about the final thermal pulses. The spectra of PNe are usually a composite, a mixture from the nebula and the illuminating central star. Many PNe are sufficiently large or bright that the nebular spectrum can be isolated.

Accurate abundances from $\mathrm{PNe}$ for helium, $\mathrm{C}, \mathrm{N}, \mathrm{O}, \mathrm{Ne}, \mathrm{S}, \mathrm{Cl}$ and $\mathrm{Ar}$ have been available for some time [2]. These abundances can be used as a powerful tool to constrain models of AGB stars [3], in particular the amount of mixing that occurs during the third dredge-up following a thermal pulse (TP). The discovery of lines of the light neutron-capture element germanium in PNe [4] has been followed in recent times by the detection of more heavy elements $(\mathrm{Ga}, \mathrm{Kr}, \mathrm{Se}, \mathrm{Xe})$ created by neutron capture [5-7]. These observations have revealed abundances from solar to $\sim 3$ to 10 times solar of heavy elements, providing now not only constraints on the amount of dredge-up but also on the operation of the slow-neutron capture process occurring in AGB stars of various masses.

In this contribution, we seek to address whether detailed nucleosynthesis models of AGB stars of various masses and metallicities can reproduce the observed amount of Ge in PNe.

\section{The Numerical Method}

We compute the structure first and then perform detailed nucleosynthesis calculations in the manner described by Lugaro et al. [8]. The reaction network in the post-processing algorithm has been expanded from 74 species to 156 , to include all stable isotopes up to ${ }^{75}$ As. The postprocessing code includes time-dependent diffusive mixing in all convective zones and computes its own time step and mass mesh. We include all $\beta$-decays, and all proton, $\alpha$ and $n$ capture reactions on all species in the network, which results in a total of 1260 reaction rates.

We use a "double neutron-sink" description [9], and include two artificial species which are linked by the following reactions ${ }^{75} \mathrm{As}(n, \gamma)^{76} \mathrm{~g}$ and ${ }^{76} \mathrm{~g}(n, L)^{76} \mathrm{~g}$, where ${ }^{76} \mathrm{~g}$ replaces ${ }^{76} \mathrm{As}$, and has an initial abundance equal to the sum of solar abundances from Se to $\mathrm{Bi}$. The second artificial particle, $L$, is equivalent to counting the number of neutrons captured beyond As. The ratio $\left(L /{ }^{76} \mathrm{~g}\right)$ is a description of the neutron captured per seed nuclei and could in principle be related to the s-process distribution.

Most of the 1260 reaction rates are from the 1991 updated REACLIB Data Tables. Some proton, $\alpha$ and $n$ capture reaction rates have been updated according to the latest experimental results, see [8] for more details. We use the NACRE [10] rates for the $\mathrm{NeNa}$ and $\mathrm{MgAl}$ chains, and rates from Karakas et al. [11] for the ${ }^{22} \mathrm{Ne}(\alpha, n){ }^{25} \mathrm{Mg}$ and ${ }^{22} \mathrm{Ne}(\alpha, \gamma){ }^{26} \mathrm{Mg}$ reactions. The crosssection of the ${ }^{76} \mathrm{~g}(n, L)^{76} \mathrm{~g}$ reaction is a composite assuming a solar heavy element distribution and thermodynamic conditions appropriate for low-mass AGB stars. 
Table 1: Details of the stellar models.

\begin{tabular}{cccccccc}
\hline \hline Mass & $Z$ & TPs & $T_{\mathrm{He}}^{\max }$ & Mass $_{\text {dred }}$ & HBB? & $\mathrm{C} / \mathrm{O}$ & ${ }^{12} \mathrm{C} /{ }^{13} \mathrm{C}$ \\
\hline 3.0 & 0.02 & 26 & 302 & $8.11(-2)$ & No & 1.40 & 118.0 \\
5.0 & 0.02 & 24 & 352 & $5.03(-2)$ & Yes & 0.77 & 7.840 \\
6.5 & 0.02 & 40 & 368 & $4.70(-2)$ & Yes & 0.40 & 11.60 \\
3.0 & 0.012 & 22 & 307 & $9.09(-2)$ & No & 2.79 & 185.0 \\
6.5 & 0.012 & 51 & 369 & $6.48(-2)$ & Yes & 0.76 & 10.40 \\
1.9 & 0.008 & 19 & 278 & $1.70(-2)$ & No & 2.08 & 143.0 \\
2.5 & 0.004 & 30 & 308 & $8.17(-1)$ & No & 14.7 & 1416 \\
\hline
\end{tabular}

\section{The stellar models}

Stellar models of $1.5,3$ and $6.5 \mathrm{M}_{\odot}$ of $Z=0.012$ were computed with the revised solar abundances from Asplund, Grevesse \& Sauval [12] for comparison to the $Z=0.02$ models, with abundances from Anders \& Grevesse [13]. We assumed scaled-solar initial abundances for the $Z=0.008$ and 0.004 models.

In Table 1 we present some details of the stellar models. Similar to the $Z=0.02$ case [14], the $1.5 \mathrm{M}_{\odot}, Z=0.012$ model did not experience any third dredge-up so we do not consider it further. From Table 1 we see that the 3 and $6.5 \mathrm{M}_{\odot}, Z=0.012$ models were similar to their $Z=0.02$ counterparts. All the other low-mass AGB models became carbon stars, whereas those models with $\mathrm{HBB}$ retained an O-rich atmospheric composition $\left(5,6.5 \mathrm{M}_{\odot}\right)$.

The results presented in this study are subject to many model uncertainties including convection and mass loss, which effect both the structure and nucleosynthesis. We refer to Herwig [1] for a detailed discussion on this topic.

\section{The inclusion of a partial-mixing zone}

The inclusion of a partial mixing zone (PMZ) at the deepest extent of dredge-up will mix protons from the envelope into the He-intershell, producing a ${ }^{13} \mathrm{C}$ pocket. In the $\mathrm{PMZ}$ neutrons are liberated during the interpulse period by the reaction ${ }^{13} \mathrm{C}(\alpha, n){ }^{16} \mathrm{O}$, which can be captured by iron-seed nuclei to produce heavy elements. Observational and theoretical evidence suggests this is the dominant neutron source in low-mass AGB stars [15], however the details of how the pocket forms and its extent in mass in the He-intershell are still unknown.

The ${ }^{22} \mathrm{Ne}(\alpha, n)^{25} \mathrm{Mg}$ reaction produces a brief strong burst of neutrons in the convective pocket during a thermal pulse when the temperature exceeds $\sim 300 \times 10^{6} \mathrm{~K}$. This is probably the dominant neutron source in intermediate-mass AGB stars where the mass of the He-shell is smaller by about an order of magnitude compared to lower mass stars, reducing the importance of the ${ }^{13} \mathrm{C}$ pocket [15].

As done in previous nucleosynthesis studies $[8,15,16]$ we artificially include a PMZ of constant mass. The masses were estimated to be between $\sim 10 \%$ to $20 \%$ of the mass of the He-rich intershell. We compute models with $n o \mathrm{PMZ}$ for all masses but the $2.5 \mathrm{M}_{\odot}, Z=0.004$ model. We 
$3 \mathrm{M}_{\odot}, Z=0.012$

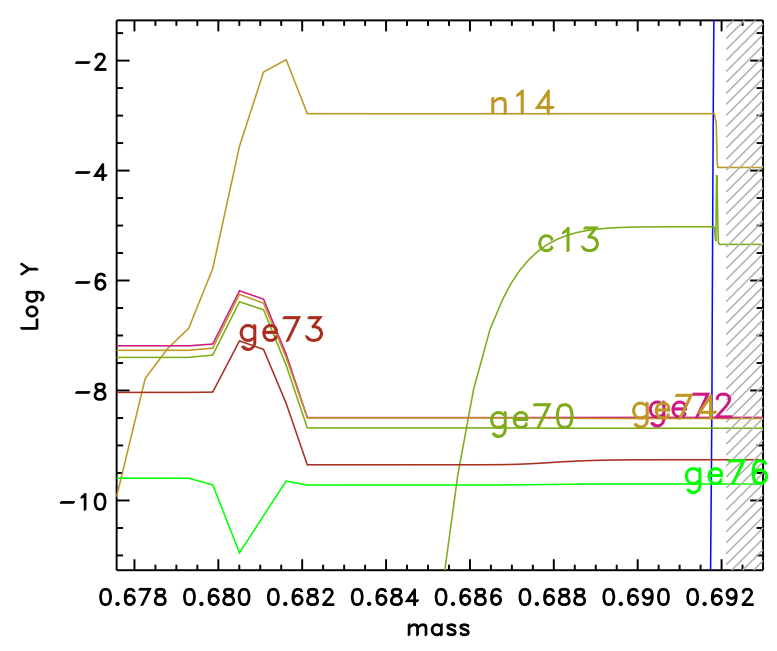

$2.5 \mathrm{M}_{\odot}, Z=0.004$

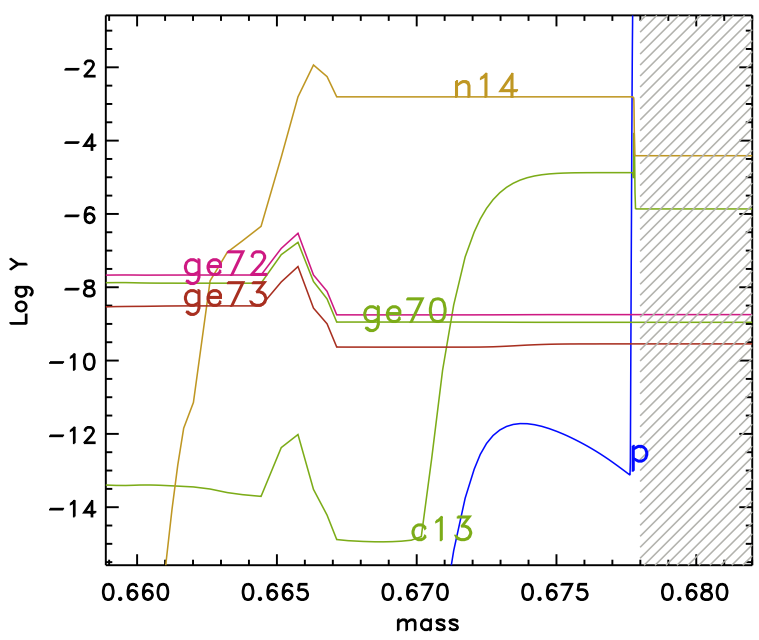

Figure 1: Composition profile showing the intershell abundances (in $\log Y$, where mass fraction $X=Y A$ ) just before the final thermal pulse. The shaded region is the inner edge of the convective envelope.

chose a proton profile in which the number of protons decreases exponentially with the mass depth below the base of the convective envelope in the same way as described by Lugaro et al. [8].

\section{Results}

The $[\mathrm{Ge} / \mathrm{Fe}]$ ratio at the surface after the last computed thermal pulse was $\lesssim 0.56$ (or a factor of $\sim 3.6$ ) for all cases but the $2.5 \mathrm{M}_{\odot}, Z=0.004$ model, where $[\mathrm{Ge} / \mathrm{Fe}]=0.77$. Models with no ${ }^{13} \mathrm{C}$ pocket produced no $\mathrm{Ge}\left(\mathrm{i} . \mathrm{e}\right.$. $[\mathrm{Ge} / \mathrm{Fe}] \approx 0$ ), except in the case of the $6.5 \mathrm{M}_{\odot}$ solar metallicity models. For these massive AGB models, where neutrons are released by efficient activation of the ${ }^{22} \mathrm{Ne}$ neutron source during thermal pulses, the increase of Ge at the tip of the TP-AGB is about a factor if 1.5 above solar $([\mathrm{Ge} / \mathrm{Fe}] \approx 0.2)$. In comparison the $5 \mathrm{M}_{\odot}, Z=0.02$ model, produced very little Ge with $([\mathrm{Ge} / \mathrm{Fe}]=0.112)$ or without $(0.04)$ a PMZ. The $2.5 \mathrm{M}_{\odot}, Z=0.004$ model produced the most Ge, partly as a consequence of the large amount of matter dredged from the core into the envelope during the TP-AGB.

Given the difficulty in producing Ge enhancements in the envelope larger than a factor of $\sim 3$, we examine intershell abundances after the last computed TP. In Figure 1 we show the abundances for a few Ge isotopes, where we see a Ge pocket left behind by the PMZ. This pocket will be diluted by intershell convection during the next pulse. The resulting abundances are: With a PMZ of $2 \times 10^{-3} \mathrm{M}_{\odot}$, we find $\mathrm{Ge}$ that is 40 times solar in the $3 \mathrm{M}_{\odot}, Z=0.012$ and $1.9 \mathrm{M}_{\odot}, Z=0.008$ models, and $\sim 60$ times solar in the $2.5 \mathrm{M}_{\odot}, Z=0.004$ case.

\section{Discussion}

Our results show that only the lower bound on the Ge abundances can be matched by con- 
sidering the envelope composition of the AGB models, with the exception of the low metallicity $2.5 \mathrm{M}_{\odot}$ case which produced a factor of 6 enhancement in Ge. Models with some remaining envelope $\left(\approx 0.7 \mathrm{M}_{\odot}\right.$ in the case of the $3 \mathrm{M}_{\odot}$ models $)$ could in principle experience further TPs and dredge-up episodes. Future work will estimate the surface enrichment from these remaining pulses in the manner described in [17].

Our results suggest that the largest Ge enhancements probably come from pure He-intershell material being mixed with a very small envelope (with little or no dilution) - this could take place during a late thermal pulse [18]. The intershell abundances produced by our models produce more than enough $\mathrm{Ge}$ ( $\gtrsim 40$ times solar) to account for the observations.

Our results compare favourably with models computed by Gallino et al. [15], where [Ge/Fe] $=0.46$ for a $3 \mathrm{M}_{\odot}, Z=0.02$ model (corresponding to a dilution of 1 part of He-intershell material per 26 parts of envelope material). The other light s-process element that we include and that has been detected in $\mathrm{PNe}$ is $\mathrm{Ga}$, with hints from observations that the Ga abundance is enhanced and follows Ge [5]. Our results and Gallino et al.s [15] approximately follow this trend where our models predict $\mathrm{Ga}$ is slightly more enhanced than Ge.

Further work will extend the nuclear network to cover the first s-process peak (up to $\mathrm{Sr}$ ), and remove many of the charged-particle reactions on heavy species not relevant for AGB star nucleosynthesis.

\section{References}

[1] F. Herwig. Evolution of Asymptotic Giant Branch Stars, ARA\&A 43 435, 2005

[2] M. A. Dopita, et al., Hubble Space Telescope Observations of Planetary Nebulae in the Magellanic Clouds. V. Mass Dependence of Dredge-up and the Chemical History of the Large Magellanic Cloud ApJ 474 188, 1997

[3] A. I. Karakas and J. C. Lattanzio, AGB Stars and the Observed Abundance of Neon in Planetary Nebulae PASA 20 393, 2003

[4] N. C. Sterling, H. L. Dinerstein, and C. W. Bowers. Discovery of Enhanced Germanium Abundances in Planetary Nebulae with the Far Ultraviolet Spectroscopic Explorer ApJL 578 L55, 2002

[5] N. C. Sterling and H. L. Dinerstein, UV Observations of Neutron Capture Elements in Planetary Nebulae Revista Mexicana de Astronomia y Astrofisica Conference Series 18 133, 2003

[6] N. C. Sterling and H. L. Dinerstein, The Abundances of Light Neutron-Capture Elements in Planetary Nebulae astro-ph/0605059,2006

[7] Y. Zhang, et al., Abundances of s-process elements in planetary nebulae: $\mathrm{Br}, \mathrm{Kr}$ and Xe astro-ph/0605181, 2006

[8] M. Lugaro, et al., Reaction Rate Uncertainties and the Production of 19F in Asymptotic Giant Branch Stars ApJ 615 934, 2004

[9] F. Herwig, N. Langer, and M. Lugaro, The s-Process in Rotating Asymptotic Giant Branch Stars ApJ $\mathbf{5 9 3} 1056,2003$

[10] C. Angulo, et al., A compilation of charged-particle induced thermonuclear reaction rates Nucl. Phys. A 656 3, 1999 
[11] A. Karakas, et al., The Uncertainties in the 22Ne + alpha-capture Reaction Rates and the Production of the Heavy Magnesium Isotopes in Asymptotic Giant Branch Stars of Intermediate Mass ApJ 643 741,2006

[12] M. Asplund, N. Grevesse, and A. J. Sauval, The Solar Chemical Composition, in proceedings of ASP Conf. Ser. 336: Cosmic Abundances as Records of Stellar Evolution and Nucleosynthesis 25 astro-ph/0410214, 2004

[13] E. Anders and N. Grevesse, Abundances of the elements - Meteoritic and solar. Geochim. Cosmochim. Acta 53 197, 1989

[14] A. I. Karakas, J. C. Lattanzio, and O. R. Pols. Parameterising the Third Dredge-up in Asymptotic Giant Branch Stars PASA 19 515, 2002

[15] R. Gallino, et al., Evolution and Nucleosynthesis in Low-Mass Asymptotic Giant Branch Stars. II. Neutron Capture and the s-Process ApJ 497 388, 1998

[16] S. Goriely and N. Mowlavi, Neutron-capture nucleosynthesis in AGB stars A\&A 362 599, 2000

[17] A. I. Karakas and J. C. Lattanzio, Production of Aluminium and the Heavy Magnesium Isotopes in Asymptotic Giant Branch Stars. PASA 20 279, 2003

[18] T. Blöcker, Evolution on the AGB and beyond: on the formation of H-deficient post-AGB stars Ap\&SS 275 1, 2001 